\title{
Nonvanishing Local Scalar Invariants even in VSI Spacetimes with all Polynomial Curvature Scalar Invariants Vanishing
}

\author{
Don N.Page* \\ Theoretical Physics Institute \\ Department of Physics, University of Alberta \\ Room 245B1 CEB, 11322 - 89 Avenue \\ Edmonton, Alberta, Canada T6G $2 G^{7}$
}

(Dated: 2009 January 28)

\begin{abstract}
VSI ('vanishing scalar invariant') spacetimes have zero values for all total scalar contractions of all polynomials in the Riemann tensor and its covariant derivatives. However, there are other ways of concocting local scalar invariants (nonpolynomial) from the Riemann tensor that need not vanish even in VSI spacetimes, such as Cartan invariants. Simple examples are given that reduce to the squared amplitude for a linearized monochromatic plane gravitational wave. These nonpolynomial local scalar invariants are also evaluated for non-VSI spacetimes such as Schwarzschild and Kerr and are estimated near the surface of the earth. Similar invariants are defined for null fluids and for electromagnetic fields.
\end{abstract}

PACS numbers: PACS 04.20.-q, 04.30.-w, 04.70.Bw, 04.20.Jb

\section{INTRODUCTION}

VSI or 'vanishing scalar invariant' spacetimes have recently been discussed [1, 2, 3, 4, [5, 6, 7, 8, 9] and defined 2] as those in which "all of the scalar invariants constructed from the Riemann tensor and its covariant derivatives are zero." This definition has the implicit assumption that 'constructed' means by "contraction of a polynomial in the Riemann tensor and its covariant derivatives" [1].

However, there are other ways of forming local scalar invariants, invariants under all passive diffeomorphisms, as defined at Eq. (1.1) of [10]. For example [11, 12, 13, 14, 15, 16, 17, 18, 19, 20, 21], there are the Cartan invariants, which are components of the Riemann tensor and its covariant derivatives ('curvature tensors') in a frame sufficiently determined by such curvature tensors that these components are invariant under any remaining freedom in the frame. (One might say that these other ways are 'concoctions,' in contradistinction to the limitations of 'constructions' as implicitly defined above.)

Here I shall give simple examples from a null vector direction determined by curvature tensors (which can be one of the null basis vector directions of a null frame given by the Cartan method, so my examples can also be obtained by that general method). The first examples I shall give are local numerical invariants $\mathcal{N}_{1}$ and $\mathcal{N}_{2}$ that are rather simply defined for generic spacetimes (though often are either zero or infinite). These are generically nonzero for at least certain classes of VSI spacetimes, such as plane gravitational waves. These examples are concocted by taking the minimum and the maximum, over variations of an auxiliary null vector, of the ratio of two scalar monomials each formed by the contraction of

*Electronic address: don@phys.ualberta.ca copies of the Riemann tensor or of its covariant derivatives and eight copies of the auxiliary null vector. For a linearized monochromatic plane gravitational wave, both of these local scalar invariants are the same and are equal to the sum of the squares of the wave amplitudes for the two polarizations, $h_{+}^{2}+h_{\times}^{2}$. In this case the average value of $\mathcal{N}_{1}=\mathcal{N}_{2}$ may also be interpreted as eight times the number of gravitons per wavelength along the direction of the wave and per Planck perpendicular area.

The values of these invariants are also analyzed in the Schwarzschild and Kerr spacetimes, even though these are not VSI spacetimes and do have nonzero scalar polynomial invariants, such as the Kretschmann invariant. In these non-VSI spacetimes, the nonpolynomial invariants $\mathcal{N}_{1}$ and $\mathcal{N}_{2}$ cannot be interpreted as the sum of squared of gravitational wave amplitudes or as the number of gravitons per wavelength and per perpendicular area.

Other examples are given that can be nonzero for null fluids. A third pair is exhibited that are generically nonzero for plane electromagnetic waves and give the number of photons per wavelength and per perpendicular area for monochromatic waves.

\section{FIRST TWO INVARIANTS}

For the first pair of invariants, $\mathcal{N}_{1}$ and $\mathcal{N}_{2}$, define the covariant quantities

$$
\begin{gathered}
\nu(\mathbf{k}) \equiv R_{a j b}^{i} R_{c i d}^{j} k^{a} k^{b} k^{c} k^{d} \\
\delta(\mathbf{k}) \equiv R_{a j b ; c d}^{i} R^{j}{ }_{e i f ; g h} k^{a} k^{b} k^{c} k^{d} k^{e} k^{f} k^{g} k^{h}
\end{gathered}
$$

which for a given event in a given spacetime are functions of an auxiliary null vector $\mathbf{k}$ with contravariant compo- 
nents $k^{a}$. Then define the $\mathbf{k}$-dependent local fraction

$$
\begin{aligned}
f(\mathbf{k}) & \equiv \frac{[\nu(\mathbf{k})]^{2}}{2 \delta(\mathbf{k})} \\
& \equiv \frac{R_{a j b}^{i} R^{j}{ }_{c i d} R_{e n f}^{m} R_{g m h}^{n} k^{a} k^{b} k^{c} k^{d} k^{e} k^{f} k^{g} k^{h}}{2 R^{i}{ }_{a j b ; c d} R^{j}{ }_{\text {eif } ; g h} k^{a} k^{b} k^{c} k^{d} k^{e} k^{f} k^{g} k^{h}}
\end{aligned}
$$

Because there are the same number of null vector components in the numerator as in the denominator, the ratio $f(\mathbf{k})$ is invariant under rescaling $\mathbf{k}$, so it only depends upon the direction of $\mathbf{k}$ over the unit sphere of directions on the null cone. Assuming that the denominator is generically nonzero for an arbitrary $\mathbf{k}$, the fact that the unit sphere is compact implies that the ratio has a minimum, which is defined to be the value of $\mathcal{N}_{1}$. If (but not only if) the denominator is nonzero for all nonzero $\mathbf{k}$ at a given event, then the ratio also has a maximum, which is defined to be the value of $\mathcal{N}_{2}$. That is, one may define the two local scalar invariants to be

$$
\mathcal{N}_{1} \equiv \min _{\mathbf{k}} f(\mathbf{k}), \mathcal{N}_{2} \equiv \max _{\mathbf{k}} f(\mathbf{k})
$$

If in $D$ dimensions one goes to a frame in which $\mathbf{k}$ is one of two null basis vectors and the remaining $D-2$ basis vectors are orthonormal spatial vectors orthogonal to both of the null basis vectors, then in both Eqs. (2.1) and (2.2) the nonzero terms in the Einstein summation over the indices $i$ and $j$ are restricted to these spatial vectors, so that both $\nu(\mathbf{k})$ and $\delta(\mathbf{k})$ are sums of perfect squares. Since each tensor in the product is symmetric in $i$ and $j$, there are generically $(D-2)(D-1) / 2$ independent squares in each sum. This is larger than the $D-2$ parameters for the direction of $\mathbf{k}$ for $D \geq 4$, so generically in spacetimes with four or more directions, both the numerator and the denominator of the ratio $f(\mathbf{k})$ are positive for all nonzero $\mathbf{k}$. This allows both $\mathcal{N}_{1}$ and $\mathcal{N}_{2}$ to be defined as finite positive local scalar curvature invariants for generic spacetimes of at least four dimensions.

However, in a spacetime that solves the vacuum Einstein equations, with the Ricci tensor $R_{a b}$ being either zero (the case with no cosmological constant) or proportional to the metric (when the cosmological constant is nonzero), the trace of each tensor in the product in either $\nu(\mathbf{k})$ or $\delta(\mathbf{k})$ is zero when $i$ is set equal to $j$ and summed, so then there are only $[(D-2)(D-1) / 2]-1$ independent squares in each sum, which equals the $D-2$ number of directional parameters of $\mathbf{k}$ for $D=4$. Therefore, in a four-dimensional vacuum spacetime, generically both the numerator and the denominator of the ratio $f(\mathbf{k})$ can be zero for discrete directions of $\mathbf{k}$. Indeed, if $\mathbf{k}$ is one of the principal null directions (PNDs) of the Weyl tensor, which always exist in four dimensions, then the numerator will vanish [22]. Unless the denominator also happens to vanish for the same null direction, one gets $\mathcal{N}_{1}=0$, the generic case for a vacuum four-dimensional spacetime. On the other hand, there may or may not be null directions for which the denominator vanishes in a vacuum four-dimensional spacetime. Both possibilities are generic, since the number of equations to be solved, 2 , matches the number of parameters for null directions, so that one can have generic vacuum spacetimes, or regions of a spacetime, in which $\mathcal{N}_{2}$ is infinite, and other generic vacuum spacetimes or regions in which $\mathcal{N}_{2}$ is finite.

In a higher-dimensional vacuum spacetime [22], the numerator will vanish if and only if $\mathbf{k}$ is a Weyl aligned null direction (WAND) [4, 23, 24, 25], the generalization of a PND to higher directions. However, for $D>4$, only algebraically special spacetimes have WANDs, so generically even in vacuum the numerator of $f(\mathbf{k})$ will never vanish, and neither will the denominator.

One may also note that in a coordinate system in which the coordinates all have the dimension of length, so that the metric components are all dimensionless, the combinations of components of the Riemann tensor and its covariant derivatives in both the numerator and denominator of $f(\mathbf{k})$ have the dimensions of length to the negative eighth power, so the ratio $f(\mathbf{k})$, and hence $\mathcal{N}_{1}$ and $\mathcal{N}_{2}$, are dimensionless, pure numerical quantities without any units. Generically when they are neither zero nor infinite, their magnitudes give two measures of the strength of the gravitational field, increasing for stronger gravitational fields. However, even in arbitrarily weak gravitational fields, it is possible to have locations in spacetime where the denominator, $2 \delta(\mathbf{k})$, of the fraction $f(\mathbf{k})$, for all $\mathbf{k}$, is always anomalously small (or even zero) in comparison with the numerator, $[\nu(\mathbf{k})]^{2}$, leading to large (or even infinite) values for $\mathcal{N}_{1}$. As noted above, $\mathcal{N}_{2}$ has greater possibilities of being infinite, since it diverges at any location where the denominator vanishes for some $\mathbf{k}$ for which the numerator does not vanish. Therefore, $\mathcal{N}_{1}$ and $\mathcal{N}_{2}$ are not always good measures of the strength of the gravitational field, as they can be arbitrarily large or even undefined (infinite), but generically they are finite and small for weak gravitational fields.

\section{PLANE GRAVITATIONAL WAVES}

Plane gravitational waves are one class of fourdimensional vacuum spacetimes which generically give nonzero $\mathcal{N}_{1}$. In fact, Malcolm MacCallum has suggested 22] that Type N spacetimes, with all principal null directions coinciding, may be the only such examples ("exercise for the readers!"). They are also examples of VSI spacetimes, with all scalar polynomial curvature invariants vanishing, even though it has long been known [12, 22] that they have nonzero Cartan curvature invariant scalars.

A general plane gravitational wave that is an exact solution of the vacuum Einstein equations has the metric [26, 27] that can be put into the form

$$
d s^{2}=-d u d v+d x^{2}+d y^{2}+\left[\left(x^{2}-y^{2}\right) h_{+}^{\prime \prime}(u)+2 x y h_{\times}^{\prime \prime}(u)\right] d u^{2},
$$

where $h_{+}(u)$ and $h_{\times}(u)$ are the wave amplitude functions for the two polarizations, functions only of the null coordinate $u$, and where the prime denotes a derivative with 
respect to that null coordinate. Geodesics in this metric obey the equations

$$
\begin{aligned}
& x^{\prime \prime}=\left(x h_{+}^{\prime \prime}+y h_{\times}^{\prime \prime}\right), \\
& y^{\prime \prime}=\left(-y h_{+}^{\prime \prime}+x h_{\times}^{\prime \prime}\right) .
\end{aligned}
$$

When the wave amplitudes are small, there exists a congruence of geodesics in which, for some period of time, the transverse coordinates $x$ and $y$ have the approximate form

$$
\begin{aligned}
& x(u) \approx x_{0}+x_{0} h_{+}(u)+y_{0} h_{\times}(u), \\
& y(u) \approx y_{0}-y_{0} h_{+}(u)+x_{0} h_{\times}(u),
\end{aligned}
$$

where $x_{0}$ and $y_{0}$ are constants for each geodesic in the congruence. These obviously obey Eqs. (3.2) when the small deviations from $\left(x_{0}, y_{0}\right)$ are dropped on the right hand sides of Eqs. (3.2). (However, including the deviations of $x$ and $y$ on the right hand sides leads to long-term average accelerations of $x$ and $y$ toward the origin as a result of the gravitational attraction of the energy contained in the gravitational wave.)

The behavior of this congruence of geodesics illustrates how $h_{+}(u)$ and $h_{\times}(u)$ are the dimensionless amplitudes for the waves. For example, if one has two particles in the congruence that are separated purely in the $x$-direction, their proper separation is $\Delta x \approx$ $\Delta x_{0}\left(1+h_{+}(u)\right)$, which oscillates as an oscillating wave goes by from the $h_{+}$polarization. Similarly, two particles separated purely in the $y$ direction have proper separation $\Delta y \approx \Delta y_{0}\left(1-h_{+}(u)\right)$, which oscillates $180^{\circ}$ out of phase from the same $h_{+}$polarization. On the other hand, two particles separated along a line bisecting the $x$ - and $y$-axes, say with $\Delta x_{0}=\Delta y_{0}$, have proper separation $(\Delta x+\Delta y) / \sqrt{2} \approx\left[\left(\Delta x_{0}+\Delta y_{0}\right) / \sqrt{2}\right]\left(1+h_{\times}(u)\right)$ from the other polarization.

For the exact plane wave metric Eq. (3.1), one can readily calculate that the nonzero components of the Riemann tensor are

$$
\begin{aligned}
& R_{\text {uxux }}=-R_{\text {uyuy }}=h_{+}^{\prime \prime}(u), \\
& R_{\text {uxuy }}=+R_{\text {uyux }}=h_{\times}^{\prime \prime}(u),
\end{aligned}
$$

plus those related by the symmetries $R_{a b c d}=R_{[a b][c d]}$. Furthermore, the only nonzero covariant derivatives of the Riemann tensor are those with respect to $u$, which are the same as the partial derivatives with respect to $u$. As a result, one can quickly calculate that the numerator of the ratio $f(\mathbf{k})$ in Eq. (2.3) is $4\left(h_{+}^{\prime \prime 2}+h_{\times}^{\prime \prime 2}\right)^{2}\left(k^{u}\right)^{8}$, and the denominator is $4\left(h_{+}^{\prime \prime \prime \prime}+h_{\times}^{\prime \prime \prime \prime}\right)\left(k^{u}\right)^{8}$. Therefore, the ratio $f(\mathbf{k})$ is independent of the auxiliary null vector $\mathbf{k}$ (though it is strictly speaking undefined if one takes $\mathbf{k}=\partial / \partial v$, the propagation null vector of the wave, which has $k^{u}=0$, but one can easily avoid that special direction for $\mathbf{k}$ ). One immediately gets that both local scalar numerical invariants are

$$
\mathcal{N}_{1}=\mathcal{N}_{2}=\frac{\left(h_{+}^{\prime \prime 2}+h_{\times}^{\prime \prime 2}\right)^{2}}{h_{+}^{\prime \prime \prime \prime}+h_{\times}^{\prime \prime \prime 2}}
$$

MacCallum has informed me 22] that this value is the reciprocal of the squared modulus of a Cartan invariant calculated by Jan Åman and put into the CLASSI 28] file wave2.nul on June 9, 1986. So this invariant is not new, but its presentation as a simple function defined for generic spacetimes may be.

If both $h_{+}(u)$ and $h_{\times}(u)$ are monochromatic sinusoidal functions with the same frequency, e.g.,

$$
\begin{aligned}
h_{+}(u) & =A_{+} \cos \left(\omega u+\phi_{+}\right), \\
h_{\times}(u) & =A_{\times} \cos \left(\omega u+\phi_{\times}\right),
\end{aligned}
$$

then one simply has

$$
\mathcal{N}_{1}=\mathcal{N}_{2}=h_{+}^{2}+h_{\times}^{2},
$$

the sum of the two $u$-dependent oscillating squared amplitudes for the two polarizations of the monochromatic plane wave.

For linearized plane waves, the effective average energy density of the waves, in a local Lorentz frame in which the null coordinate of propagation of the waves is $u=t-z$, is [29], in units with $G=c=1$, the average of $\left(h_{+}^{\prime 2}+\right.$ $\left.h_{\times}^{\prime 2}\right) /(16 \pi)$, which for monochromatic waves of frequency $\omega$ is the average of $\omega^{2}\left(h_{+}^{2}+h_{\times}^{2}\right) /(16 \pi)=\mathcal{N}_{1} \omega^{2} /(16 \pi)$. Over one wavelength $2 \pi / \omega$ in the $z$-direction, the average energy per transverse area (in the $x-y$ plane) is thus the average of $\mathcal{N}_{1} \omega / 8$ over one wavelength. Then since each graviton of the monochromatic wave has energy $\omega$ in units with $\hbar=1$, in Planck units $(\hbar=c=G=1)$ the number of gravitons per wavelength and per transverse area is simply the average of $\mathcal{N}_{1} / 8$. That is, the value of $\mathcal{N}_{1}=\mathcal{N}_{2}$ averaged over one wavelength of a monochromatic linearized plane gravitational wave is eight times the number of gravitons per wavelength and per transverse area.

Although the number of gravitons per wavelength and per transverse area is a Lorentz-invariant quantity in the case of a uniform flux of monochromatic gravitons all traveling in the same direction, this number is a nonlocal quantity (since the gravitons are not localized). However, for classical gravitational fields, the numerical scalar invariants $\mathcal{N}_{1}$ and $\mathcal{N}_{2}$ defined by Eq. (2.4) have the advantage of being purely local. They are well defined for all locations where the denominator of $f(\mathbf{k})$ in Eq. (2.3) is nonzero for all possible auxiliary null vectors $\mathbf{k}$, and also when the denominator vanishes for some particular null vectors provided that the numerator also vanishes just as fast at those null vectors (as in the plane wave example above).

One can have situations in which the numerical scalar invariants $\mathcal{N}_{1}$ and $\mathcal{N}_{2}$ are not defined (or are infinite), if the denominator of the ratio $f(\mathbf{k})$ given in Eq. (2.3) is zero for all possible auxiliary null vectors $\mathbf{k}$ (e.g., in de Sitter) for $\mathcal{N}_{1}$ or goes to zero faster than the numerator does as one approaches some special null vectors for $\mathcal{N}_{2}$. (The fact that $\mathcal{N}_{1}$ is defined as the minimum of the ratio means that if the denominator, which is always nonnegative, is nonzero for any $\mathbf{k}, \mathcal{N}_{1}$ will be finite, though $\mathcal{N}_{2}$, 
the maximum of the ratio $f(\mathbf{k})$, would be infinite at any location where the denominator is zero while the numerator remains nonzero for some $\mathbf{k}$, or where both go to zero but the denominator goes to zero faster as $\mathbf{k}$ approaches a value where the denominator vanishes.)

For the plane waves considered above, this vanishing of the denominator for all $\mathbf{k}$ is indeed the case if one has $h_{+}^{\prime \prime \prime \prime 2}+h_{\times}^{\prime \prime \prime \prime 2}=0$, where the 4 th derivatives of both polarization amplitudes vanish. If one has a monochromatic wave, the numerator would also vanish at the same values of $u$, so that one would get a finite limit of the ratio (actually zero in the monochromatic case) if one approached it from positions where the denominator did not vanish, but if one has a non-monochromatic wave, one can have nongeneric situations in which $h_{+}^{\prime \prime \prime \prime 2}+h_{\times}^{\prime \prime \prime \prime 2}$ vanishes but $h_{+}^{\prime \prime 2}+h_{\times}^{\prime \prime 2}$ remains nonzero, which would make $\mathcal{N}_{1}$ and $\mathcal{N}_{2}$ infinite. For example, one could have $h_{+}(u)=h_{\times}(u)=$ $16 A \cos (\omega u)-A \cos (2 \omega u)$ at $\omega u=2 \pi n$ for any integer $n$ (hypersurfaces of measure zero in the entire spacetime). Or, one could have a very special spacetime in which, say, $h_{+}(u)=h_{\times}(u)=B u^{2}$, which would make $\mathcal{N}_{1}$ and $\mathcal{N}_{2}$ infinite everywhere. However, for a generic position in a generic nonvacuum spacetime of at least four dimensions (as well as for at least the plane wave VSI spacetimes), $\mathcal{N}_{1}$ and $\mathcal{N}_{2}$ are well defined and are both nonzero and finite. (For generic vacuum spacetime in four dimensions, $\mathcal{N}_{1}$ is zero, and $\mathcal{N}_{2}$ may be either finite or infinite.)

\section{SCHWARZSCHILD METRIC}

For the Schwarzschild metric, the numerator, $[\nu(\mathbf{k})]^{2}$, of $f(\mathbf{k})$ given in Eqs. (2.1) and (2.3) is fairly straightforward to evaluate by hand (though it did take me several hours to calculate it, check it, and correct a mistake I made originally), but the denominator appeared to be beyond my ability to calculate by hand in any reasonable time. Therefore, I used GRTensorII Version 1.79 (R4) 30], which also took me several hours, with help from James MacKinnon and Andrei Zelnikov, to install it and learn the basics of using it, so it would not have been worth the effort just for the numerator alone. GRTensor enabled me to find that the numerator was exactly as calculated by hand ( 0.07 seconds CPU time) and to calculate the denominator (44.67 seconds CPU time, over 600 times as long).

Initially I did the calculation in the standard orthonormal frame outside the event horizon, with the spatial part of the null vector making an angle $\alpha$ with the radial direction in a local Lorentz frame of an observer at rest with respect to the black hole. After some simplification by hand of the result from GRTensor, I got

$$
f(\mathbf{k})=\left[\frac{6 M}{5 r} \frac{1}{1+\left(1-\frac{2 M}{r}\right)\left(12 \csc ^{2} \alpha-15\right)}\right]^{2} .
$$

To get $f(\mathbf{k})$ both inside and outside the event horizon, it is better to use ingoing Eddington-Finkelstein [29, 31,
32] coordinates, in which the Schwarzschild metric is

$$
d s^{2}=-\left(1-\frac{2 M}{r}\right) d v^{2}+2 d v d r+r^{2} d \theta^{2}+r^{2} \sin ^{2} \theta d \phi^{2} .
$$

Then I used the one-parameter family of null vectors at each location,

$$
\mathbf{k}=\partial_{v}+\frac{1}{2}\left(1-\frac{2 M}{r}-x^{2}\right) \partial_{r}+\frac{x}{r} \partial_{\theta},
$$

where $x$ is the one parameter (sufficient for a generic null vector for Eq. (2.3), because of the spherical symmetry of the metric and because of the independence of $f(\mathbf{k})$ on the magnitude of the null vector $\mathbf{k}$ ). The parameter $x$ may take any real value. Outside the horizon, $x=$ $\sqrt{1-2 M / r} \cot (\alpha / 2)$, but the null vector $\mathbf{k}$ given by Eq. (4.3) is well defined for any real $x$ even on and inside the horizon, i.e., for all $r>0$.

With this form of the Schwarzschild metric and of the null vector, the hand-simplified result of GRTensor for the fraction $f(\mathbf{k})$ defined by Eq. (2.3) is

$$
f(\mathbf{k})=\left(\frac{2 M}{5 r}\right)^{2}\left[x^{2}-\left(\frac{8}{3}-\frac{6 M}{r}\right)+\left(1-\frac{2 M}{r}\right)^{2} \frac{1}{x^{2}}\right]^{-2}
$$

One can readily check that outside the horizon, using the relation given in the previous paragraph between the parameters $x$ and $\alpha$, that $f(\mathbf{k})$ given by Eq. (4.1) agrees with $f(\mathbf{k})$ given by Eq. (4.4).

The fraction $f(\mathbf{k})$ given by Eq. (4.4), a perfect square for the Schwarzschild metric, goes to zero when $x$ is taken to infinity (and also when $x$ is taken to zero at all locations except at the event horizon, $r=2 M)$. Therefore, for all locations in the Schwarzschild metric,

$$
\mathcal{N}_{1} \equiv \min _{\mathbf{k}} f(\mathbf{k})=0 \text {. }
$$

This vanishing of $\mathcal{N}_{1}$ appears to be a generic feature of four-dimensional vacuum metrics, as we have seen above.

On the other hand, $\mathcal{N}_{2}$, the maximum of $f(\mathbf{k})$ over all null vectors $\mathbf{k}$, depends on the location. For $r \geq 3 M$, $f(\mathbf{k})=\infty$ at

$$
x^{2}=\frac{4}{3}-\frac{3 M}{r} \pm \sqrt{\left(\frac{1}{3}-\frac{M}{r}\right)\left(\frac{7}{3}-\frac{5 M}{r}\right)},
$$

so for $r \geq 3 M$,

$$
\mathcal{N}_{2} \equiv \max _{\mathbf{k}} f(\mathbf{k})=\infty
$$

For $r \leq 3 M$, the maximum for $f(\mathbf{k})$ is at

$$
x^{2}=\left|1-\frac{2 M}{r}\right| \text {, }
$$

so for $2 M \leq r \leq 3 M$,

$$
\mathcal{N}_{2} \equiv \max _{\mathbf{k}} f(\mathbf{k})=\left[\frac{3 M}{5(3 M-r)}\right]^{2},
$$


whereas for $0<r \leq 2 M$,

$$
\mathcal{N}_{2} \equiv \max _{\mathbf{k}} f(\mathbf{k})=\left[\frac{3 M}{5(15 M-7 r)}\right]^{2} .
$$

At the horizon itself $(r=2 M), \mathcal{N}_{2}=9 / 25=0.36$.

In summary, for the Schwarzschild metric, $\mathcal{N}_{1}$, the minimum at each location, over all null vectors $\mathbf{k}$, of $f(\mathbf{k})$ given by Eq. (2.3), is zero everywhere, whereas $\mathcal{N}_{2}$, the maximum of $f(\mathbf{k})$, is infinite for $r \geq 3 M$ but is finite for $r<3 M$, given by Eqs. (4.9) and (4.10) immediately above for $2 M \leq r \leq 3 M$ and $0<r \leq 2 M$ respectively.

\section{KERR METRIC}

For the Kerr metric with an orthonormal null basis $(\mathbf{l}, \mathbf{n}, \mathbf{m}, \overline{\mathbf{m}})$ aligned along the eigendirections of the Weyl tensor and normalized so that $\mathbf{l} \cdot \mathbf{n}=-1$ and $\mathbf{m} \cdot \overline{\mathbf{m}}=+1$ (and with all other scalar products vanishing), and with the one-complex-parameter $(z)$ family of auxiliary null vectors at each location being

$$
\mathbf{k}=\mathbf{l}+z \bar{z} \mathbf{n}+z \mathbf{m}+\bar{z} \overline{\mathbf{m}}
$$

one may calculate by hand and by GRTensor ( 0.13 seconds CPU time) that the numerator of $f(\mathbf{k})$ given in Eq. (2.3) is

$$
\left(R_{a j b}^{i} R_{c i d}^{j} k^{a} k^{b} k^{c} k^{d}\right)^{2}=72^{2}\left|\psi_{2}\right|^{4}|z|^{8},
$$

where 33

$$
\psi_{2}=-\frac{M}{(r-i a \cos \theta)^{3}}
$$

is the only nonzero Weyl curvature scalar in the Type-D Kerr metric.

My unskilled efforts with GRTensor were unsuccessful in calculating the denominator $2 \delta(\mathbf{k})$ of $f(\mathbf{k})$, so that I gave up after my computer had run nearly twentyfour hours without getting an answer. However, Nicos Pelavas [34] and Malcolm MacCallum [22] kindly calculated the denominator for me. Pelavas' output takes up about one page of print and is a polynomial in $z$ and $\bar{z}$ with each having powers running from 2 through 6 (25 terms), and with coefficients functions of $M, a, r$, and $\theta$. As I had conjectured, the denominator is proportional to $|z|^{4}$ for small $|z|$, with a coefficient (which I could not calculate) of $2^{6} 3^{4} 5^{2} M^{-4 / 3}\left|\psi_{2}\right|^{10 / 3}$, so for small $|z|$, $f \sim 0.04\left|M^{2} \psi_{2}\right|^{2 / 3}|z|^{4}$, which has a minimum value of $\mathcal{N}_{1}=0$ at $z=0$. Thus indeed $\mathcal{N}_{1}$ vanishes for Kerr, as it is expected to do for a generic vacuum four-dimensional spacetime.

Malcolm MacCallum 22] used spinor notation to show how the denominator, $2 \delta(\mathbf{k})$, can be written as the absolute square of the second covariant derivative of a Weyl curvature spinor in a frame with $\mathbf{k}$ being one of the null basis vectors. He explicitly calculated this complex second derivative and expressed it as a sum of 14 terms, a reduction from the 25 terms Pelavas had given me for its absolute square.

From MacCallum's expression, I derived the following expression for the fraction $f(\mathbf{k})$ :

$$
f(\mathbf{k})=\left|\frac{2 M \rho}{5 \Delta D}\right|^{2}
$$

where $\rho \equiv \sqrt{r^{2}+u^{2}}, u \equiv a \cos \theta, \Delta \equiv r^{2}-2 M r+a^{2}$, and

$$
\begin{aligned}
D= & \left(|z|-\frac{1}{|z|}\right)^{2}+A^{2}\left(i \sqrt{\frac{z}{\bar{z}}}-i \sqrt{\frac{\bar{z}}{z}}\right)^{2}-\frac{2}{3} \frac{P}{\rho^{2} \Delta} \\
& +\frac{2}{3} i\left[A\left(\frac{3}{z}-3 z+\frac{4}{\bar{z}}-4 \bar{z}\right)-\frac{Q}{\rho^{2} \Delta}\right] \\
= & 4 \cot ^{2} \alpha-\frac{4}{3} A \csc \alpha \sin \beta+4 A^{2} \sin ^{2} \beta-\frac{2}{3} \frac{P}{\rho^{2} \Delta} \\
& +\frac{2}{3} i\left[14 A \cot \alpha \cos \beta-\frac{Q}{\rho^{2} \Delta}\right]
\end{aligned}
$$

with $z=\tan (\alpha / 2) e^{i \beta}$, where $\alpha$ is the angle between the spatial part of $\mathbf{k}$ and the radial direction and $\beta$ is the angle between the nonradial spatial part of $\mathbf{k}$ and the $\theta$ direction in the orthonormal frame determined by the orthonormal null basis, $\mathbf{e}_{\hat{0}}=(\mathbf{l}+\mathbf{n}) / \sqrt{2}, \mathbf{e}_{\hat{r}}=(\mathbf{l}-\mathbf{n}) / \sqrt{2}$, $\mathbf{e}_{\hat{\theta}}=(\mathbf{m}+\overline{\mathbf{m}}) / \sqrt{2}, \mathbf{e}_{\hat{\phi}}=(\mathbf{m}-\overline{\mathbf{m}}) /(i \sqrt{2})$, with the null boost freedom in $\mathbf{l}$ and $\mathbf{n}$ and the rotational freedom in $\mathbf{m}$ and $\overline{\mathbf{m}}$ used to make $\mathbf{e}_{\hat{r}}$ and $\mathbf{e}_{\hat{\theta}}$ orthogonal to the Killing vectors $\partial / \partial t$ and $\partial / \partial \phi$, with

$$
A \equiv \sqrt{\frac{a^{2}-u^{2}}{\Delta}} \equiv \frac{a \sin \theta}{\sqrt{r^{2}-2 M r+a^{2}}},
$$

$P \equiv r^{4}-3 M r^{3}+8 a^{2} r^{2}-6 u^{2} r^{2}+M u^{2} r+8 a^{2} u^{2}-7 u^{4}$

and with

$$
Q \equiv M u\left(3 r^{2}-u^{2}\right) \equiv M a \cos \theta\left(3 r^{2}-a^{2} \cos ^{2} \theta\right) .
$$

The value of $\mathcal{N}_{1}$, which is zero, is the minimum value of $f(\mathbf{k})$, which corresponds to the maximum absolute value of $D$, occurring for $|z|=0(\alpha=0)$ and for $|z|=\infty$ $(\alpha=\pi)$. The value of $\mathcal{N}_{2}$ is the maximum value of $f(\mathbf{k})$, which corresponds to the minimum absolute value of $D$. For $r \gg M \geq a$ and for $\theta$ not too near either 0 or $\pi$ (the axes of rotation), one can always choose the complex $z$, or equivalently the direction angles $\alpha$ and $\beta$ for the null vector $\mathbf{k}$, so that both the real and imaginary parts of $D$ are zero. In particular, if $\cot \alpha \cos \beta$ is fixed to have the value $Q /\left(14 A \rho^{2} \Delta\right)$ to make the imaginary part of $D$ vanish, so long as this value is not too great, one can choose $\beta$ (and hence $\alpha$ ) to make the real part of $D$ vanish as well.

However, if $Q /\left(14 A \rho^{2} \Delta\right)$ is too large, then for all $\beta$ the real part of $D$ will be positive when the imaginary part is set to zero, so that $D$ can never be zero for any complex 
$z$, and $f(\mathbf{k})$ will have a finite maximum value that is $\mathcal{N}_{2}$. One can readily show that asymptotically at large $r$ the condition for $\mathcal{N}_{2}$ to be finite is $\tan \theta \lesssim \sqrt{27 / 98}(M / r)$, so that the region with finite $\mathcal{N}_{2}$ is a cylinder about the axis with asymptotic radius $\sqrt{27 / 98}$ M.

One can also show that on the axis itself, where $A=0$, and for $r$ large enough that $P>0$ (which in the limit of small $a$ is for $r>3 M$ ),

$\mathcal{N}_{2}=\frac{9\left(r^{2}+a^{2}\right)^{3}}{25 a^{2}\left(3 r^{2}-a^{2}\right)^{2}}=0.04 \frac{r^{2}}{a^{2}}\left(1+\frac{a^{2}}{r^{2}}\right)^{3}\left(1-\frac{a^{2}}{3 r^{2}}\right)^{-2}$.

\section{NEAR THE SURFACE OF THE EARTH}

If one approximated the gravitational field near the surface of the earth by the Schwarzschild metric, then $\mathcal{N}_{1}$ would be zero and $\mathcal{N}_{2}$ would be infinite. However, because of perturbations of the mass distribution from spherical symmetry, I would suspect that both the numerator and the denominator of $f(\mathbf{k})$ given in Eq. (2.3) would at generic nonvacuum locations be nonzero for all directions of the auxiliary null vector $\mathbf{k}$, so that both $\mathcal{N}_{1}$ and $\mathcal{N}_{2}$ would be nonzero and finite. (For vacuum locations, after the insights given by [22], I suspect that generically $\mathcal{N}_{1}$ would be zero, whereas $\mathcal{N}_{2}$ could generically be either finite or infinite.)

One might think that a reasonably good estimate for $f(\mathbf{k})$ and for its minimum $\left(\mathcal{N}_{1}\right)$ and maximum $\left(\mathcal{N}_{2}\right)$ values at some location would be obtained by taking the monopole plus quadrupole contributions to the Newtonian gravitational field of the earth. That might indeed be correct for the numerator of $f(\mathbf{k})$ when one is far above the surface of the earth and its higher multipole irregularities, at least if one ignores the contribution from the gravitational fields of the sun and moon. However, since the denominator has each Riemann tensor with two covariant derivatives, the small-scale variations from local mass distributions are likely to give by far the largest contribution to the denominator near the surface of the earth. They are also likely to prevent the numerator from having a minimum (say with the timelike part of the null vector fixed) nearly so small as it would be from just the quadrupole field of the earth.

For concreteness, let us fix the magnitude of the auxiliary null vector $\mathbf{k}$ so that its timelike part is unity in the frame of the surface of the earth (i.e., so that the dot product of the null vector with the four-velocity of the earth surface is -1 ). Then in this same frame, the Riemann curvature tensor will have typical components of the order of magnitude of

$$
C=\frac{G M_{\oplus}}{R_{\oplus}^{3} c^{2}} \approx 1.7 \times 10^{-23} \mathrm{~m}^{-2},
$$

where $M_{\oplus}$ and $R_{\oplus}^{3} c^{2}$ are the mass and radius of the earth. Nearby objects that distort the spherical gravitational field will give distortions in the Riemann tensor of the same order of magnitude (more nearly correctly, smaller by the ratios of the densities of the objects to the average density of the earth, but since these ratios are of the order of unity, here I shall ignore them).

Therefore, the magnitude of the numerator of $f(\mathbf{k})$ can be expected to be of the order of $C^{4}$ for generic directions of the spacelike part of $\mathbf{k}$. From the results above for the Schwarzschild metric, it would vary as $\sin ^{8} \alpha$, where $\alpha$ is the angle between the spatial part of the null vector and the radial direction, if the gravitational field were precisely spherical and vacuum, but the distortions of nearby objects, e.g., in one's office, would presumably make the numerator have a magnitude of the order of $C^{4}$ for generic directions of the spacelike part of $\mathbf{k}$. However, in a generic vacuum spacetime, the numerator would be expected to vanish as $\sin ^{4} \alpha$, where $\alpha$ is now the angle between the spatial part of the null vector and the spatial part of the nearest principal null direction. In a non-vacuum region, the minimum value of the numerator (with the magnitude of $\mathbf{k}$ fixed as above) would be expected to be of the order of the tracefree part of the Ricci tensor, $G \rho / c^{2} \sim C \rho / \rho_{\oplus}$, where $\rho$ is the mass density of the location and $\rho_{\oplus}$ is the average mass density of the earth.

Similarly, the magnitude of the denominator of $f(\mathbf{k})$ can be expected to be of the order of $C^{2} / L^{4}$ for generic directions of the spacelike part of $\mathbf{k}$, where $L$ is a typical length scale for the smallest nearby object (at a distance comparable to its size) that is distorting the Riemann tensor of the earth's gravitational field. Therefore, one would expect that $f(\mathbf{k})$ would for generic directions of the spacelike part of $\mathbf{k}$ have magnitudes of the order of $C^{2} L^{4}$, with its maximum value being of this same order (if it is finite) and its minimum value being of the order of $C^{2} L^{4} \rho / \rho_{\oplus}$. If one takes $L \sim 1 \mathrm{~m}$, say for the old CRT computer monitor some fraction of a meter in front of me that I had when I started writing this paper, and a location within the air where $\rho / \rho_{\oplus} \sim 10^{-3}$, then one would expect that, very crudely,

$$
\mathcal{N}_{1} \sim 10^{-26}, \mathcal{N}_{2} \sim 10^{-23}
$$

(unless $\mathcal{N}_{2}=\infty$ ) at a typical location in the air, within a meter or so of lumpy stuff of roughly the density of the earth. These order-of-magnitude estimates exhibit the fact that for generic weak nonvacuum gravitational fields, I would expect both $\mathcal{N}_{1}$ and $\mathcal{N}_{2}$ to be very small, increasing quadratically with the strength of the field (e.g., quadratically with the mass densities and hence also with $C$ for a given spatial distribution of the relative densities).

\section{OTHER LOCAL INVARIANTS NONZERO FOR VSI SPACETIMES}

The examples of $\mathcal{N}_{1}$ and $\mathcal{N}_{2}$ defined by Eq. (2.4) as the minimum and maximum, respectively, of the ratio 
$f(\mathbf{k})$ given in Eq. (2.3), are of course only two examples of an obviously infinite sequence of such examples of local invariants that might be nonzero for VSI spacetimes. (Those examples were merely the simplest examples that I could readily think of.) All one needs is some contraction of Riemann tensors and their covariant derivatives with null vectors in both the numerator and denominator, so that there are the same number of copies of each null vector in both (in order that the ratio not depend on the normalization of each null vector, but only on its direction, which forms a compact set allowing one to take the minimum and the maximum of the ratio as local invariants). One can even have arbitrarily many different null vectors in both the numerator and denominator, so long as each null vector occurs the same number of times in both. Alternatively, one can have different numbers of null vectors in the numerator and denominator if one normalizes them so that, say, either the numerator or the denominator is fixed to be unity.

Instead of equivalence classes of null vectors related by scalar multiplication for the objects to contract with the Riemann tensors and their covariant derivatives, one could alternatively take equivalence classes of other geometric objects that are equally contracted with Riemann tensor objects in both the numerator and the denominator, at least so long as each equivalence class forms a compact set so that one can define the maximum and minimum of the resulting ratio.

Of course, it might be difficult to evaluate more complex examples. Even the simple examples given here appear rather intractable much beyond the simple cases considered above of a plane gravitational wave and of the Schwarzschild metric. However, their conceptual possibility shows that there are far more local invariants for a gravitational field that may be concocted than just the polynomial invariants that are normally constructed.

However, all of these local scalar invariants are just special cases of what can be constructed from the Cartan invariants, since they are determined by components of the curvature and of its covariant derivatives in special bases either partially or totally determined by the curvature and its derivatives. Therefore, they are not logically independent methods of concocting other invariants beyond those that may be obtained from the Cartan invariants, but some of them might be thought of as somewhat simpler concoctions that shortcut the most general form of the Cartan procedure.

It might also be worthwhile being reminded that if one does not restrict attention to local invariants, as was done here, then there would be an even vastly greater set of invariants. For example, even for flat spacetime, if space is compactified into a torus, then the periods and angles of the torus would be nonlocal invariants. When there is curvature, one can readily think of even many more ways to construct nonlocal invariants.

\section{INVARIANTS FOR NULL FLUIDS AND ELECTROMAGNETIC FIELDS}

The examples of $\mathcal{N}_{1}$ and $\mathcal{N}_{2}$ defined above by Eq. (2.4) were concocted to be nonzero for generic spacetimes, and also for generic plane waves that are VSI spacetimes, though apparently $\mathcal{N}_{1}$ is zero for generic four-dimensional vacuum spacetimes. However, one can have VSI spacetimes that are not vacuum but have null fluids, electromagnetic waves, dilatons, and/or supergravity fluxes [1, 2, 3, 4, 6, 7, 8, 9], for which in generic circumstances one can concoct nonzero local invariants that are simpler than $\mathcal{N}_{1}$ and $\mathcal{N}_{2}$.

For example, instead of the ratio $f(\mathbf{k})$ defined by Eq. (2.3), for a nonvacuum spacetime one could define

$$
\hat{f}(\mathbf{k}) \equiv \frac{R_{a b} R_{c d} k^{a} k^{b} k^{c} k^{d}}{R_{a b ; c d} k^{a} k^{b} k^{c} k^{d}}
$$

in terms of the Ricci tensor $R_{a b}$ (or in terms of its tracefree part, $S_{a b}=R_{a b}-\frac{1}{D} R g_{a b}$ in $D$ dimensions, which would give the same result) and then take

$$
\mathcal{N}_{3} \equiv \min _{\mathbf{k}} \hat{f}(\mathbf{k}), \mathcal{N}_{4} \equiv \max _{\mathbf{k}} \hat{f}(\mathbf{k})
$$

Assuming that the covariant derivatives of the Ricci tensor are suitably nonzero, these invariants may be well defined and nonzero even for generic null fluid VSI spacetimes.

For an electromagnetic field tensor $F_{a b}$, one might define

$$
\tilde{f}(\mathbf{k}) \equiv \frac{F_{a}^{i} F_{i b} F_{c}^{j} F_{j d} F_{e}^{k} F_{k f} k^{a} k^{b} k^{c} k^{d} k^{e} k^{f}}{F_{a ; b c}^{i} F_{i d ; e f} k^{a} k^{b} k^{c} k^{d} k^{e} k^{f}}
$$

and then take

$$
\mathcal{N}_{5} \equiv \min _{\mathbf{k}} \tilde{f}(\mathbf{k}), \mathcal{N}_{6} \equiv \max _{\mathbf{k}} \tilde{f}(\mathbf{k})
$$

For simplicity (though more general examples in curved spacetime would also be interesting to investigate), let us consider the case in which the electromagnetic field stress-energy tensor has a negligible effect upon the spacetime, which has the nearly flat metric

$$
d s^{2}=-d u d v+d x^{2}+d y^{2}
$$

with $u=t-z, v=t+z$. Then take the example of a plane electromagnetic wave traveling in the $z$-direction, with electromagnetic potential one-form

$$
A=A_{x}(u) d x+A_{y}(u) d y
$$

and electromagnetic field two-form

$$
F=d A=A_{x}^{\prime}(u) d u \wedge d x+A_{y}^{\prime}(u) d u \wedge d y .
$$

With $u=t-z$, this gives $E_{x}=B_{y}=-A_{x}^{\prime}$ and $E_{y}=$ $-B_{x}=-A_{y}^{\prime}$. This plane-wave electromagnetic field then gives $\tilde{f}(\mathbf{k})$ that is independent of $\mathbf{k}$ and has the form

$$
\mathcal{N}_{5}=\mathcal{N}_{6}=\frac{\left(A_{x}^{\prime 2}+A_{y}^{\prime 2}\right)^{3}}{A_{x}^{\prime \prime \prime 2}+A_{y}^{\prime \prime \prime}} .
$$


If both $A_{x}(u)$ and $A_{y}(u)$ are monochromatic sinusoidal functions with the same frequency, e.g.,

$$
\begin{gathered}
A_{x}(u)=a_{x} \cos \left(\omega u+\phi_{x}\right), \\
A_{y}(u)=a_{y} \cos \left(\omega u+\phi_{y}\right),
\end{gathered}
$$

then one has

$$
\mathcal{N}_{5}=\mathcal{N}_{6}=\left(E_{x}^{2}+E_{y}^{2}\right)^{2} / \omega^{4} .
$$

Since the energy density of the electromagnetic wave is $\left(E_{x}^{2}+E_{y}^{2}\right) /(4 \pi)$, over one wavelength $2 \pi / \omega$ in the $z$ direction the average energy per transverse area (in the $x-y$ plane) is thus the average of $\sqrt{\mathcal{N}_{5}} \omega / 2$ over one wavelength. Then since each photon of the monochromatic wave has energy $\omega$, the number of photons per wavelength and per transverse area is simply the average of $\sqrt{\mathcal{N}_{5}} / 2$. That is, the value of $\sqrt{\mathcal{N}_{5}}=\sqrt{\mathcal{N}_{6}}$ averaged over one wavelength of a monochromatic linearized plane electromagnetic wave is twice the number of photons per wavelength and per transverse area.

\section{CONCLUSIONS}

In this paper I have given simple illustrations of the long-known fact that VSI spacetimes (which have all scalar invariants vanishing everywhere that are constructed as polynomials in total contractions of the Riemann tensor and its covariant derivatives) have other classes of local scalar invariants that generically do not vanish. The examples in this paper are concocted by taking the ratio of total contractions of the Riemann tensor and its covariant derivatives with the same number of copies of an auxiliary null vector in both the numerator and denominator and then taking the minimum or the maximum of the ratio as the direction of the null vector is varied over its compact unit sphere on the null cone. When these minima or maxima are finite, they are well-defined local Lorentz invariants of the gravitational field. Examples were given that reduce to the squared amplitudes of monochromatic plane gravitational waves (and whose average over one wavelength is proportional to the number of gravitons per wavelength and per crosssectional area). The first of these examples seems to vanish for generic vacuum spacetimes in four dimensions. Both of these example local scalar invariants were fully calculated for the Schwarzschild metric, partially calculated for the Kerr metric, and estimated near the surface of the earth. Other examples were given for null fluids or null electromagnetic fields.

These examples illustrate the point made by the Car$\tan$ constructions $11,13,14,15,17,18,19,20,21]$ that local scalar invariants may be concocted that are nonzero for a larger class of spacetimes than one might naïvely think, such as the VSI spacetimes for which all scalar polynomial invariants in the curvature and its derivatives vanish.

\section{Acknowledgments}

I am grateful to Peter Musgrave, Denis Pollney and Kayll Lake [30] for providing GRTensor free of charge for calculating the first two invariants above for the Schwarzschild metric, to James MacKinnon and Andrei Zelnikov for helping me install GRTensor and use it, to Jan Åman, Alan Coley, Stanley Deser, Maciej Dunajski, Gary Gibbons, Sigbjørn Hervik, Malcolm MacCallum, Robert Milson, Georgios Papadopoulos, Nicos Pelavas, and Vojtech Pravda for extensive email discussions after the first version of this paper, and to two anonymous referees for suggesting corrections and clarifications. This research was supported in part by the Natural Sciences and Engineering Research Council of Canada. 
[1] V. Pravda, A. Pravdova, A. Coley, and R. Milson, Class. Quant. Grav. 19, 6213-6236 (2002) arXiv:gr-qc/0209024.

[2] A. A. Coley, Phys. Rev. Lett. 89: 281601 (2002) arXiv:hep-th/0211062.

[3] A. Coley, R. Milson, N. Pelavas, V. Pravda, A. Pravdova, and R. Zalaletdinov, Phys. Rev. D 67: 104020 (2003) arXiv:gr-qc/0212063.

[4] A. Coley, R. Milson, V. Pravda, and A. Pravdova, Class. Quant. Grav. 21, 5519-5542 (2004) arXiv:gr-qc/0410070.

[5] N. Pelavas, A. Coley, R. Milson, V. Pravda, and A. Pravdova, J. Math. Phys. 46, 063501 (2005) arXiv:gr-qc/0503040.

[6] A. Fuster and N. Pelavas, in Proceedings of the Eleventh Marcel Grossmann Meeting on Recent Developments in Theoretical and Experimental General Relativity, Gravitation and Relativistic Field Theories, edited by H. Kleinert, R. T. Jantzen, and R. Ruffini (World Scientific, Singapore, 2008), pp. 1302-1304 arXiv:hep-th/0611103.

[7] A. Coley, A. Fuster, S. Hervik, and N. Pelavas, Class. Quant. Grav. 23, 7431-7444 (2006) arXiv:gr-qc/0611019.

[8] A. Coley, A. Fuster, S. Hervik, and N. Pelavas, JHEP 0705, 032 (2007) arXiv:hep-th/0703256.

[9] A. A. Coley, G. W. Gibbons, S. Hervik, and C. N. Pope, Class. Quant. Grav. 25, 145017 (2008) arXiv:0803.2438 [hep-th]].

[10] J. Bicak and V. Pravda, Class. Quant. Grav. 15, 15391555 (1998) arXiv:gr-qc/9804005.

[11] E. Cartan, Leçons sur la Geometrie des Espaces de Riemann (Gauthier-Villars, Paris, 1946).

[12] W. Kundt, in Recent Developments in General Relativity (in Honour of L. Infeld) (Polish Academy of Sciences and Pergamon Press, 1962), pp. 307-311; and in Les Theories Relativistes de la Gravitation, edited by A. Lichnerowicz and M. A. Tonnelat (Colloques Internationaux du Centre National de la Recherche Scientifique, CNRS Paris, 1962), vol. XCI, p. 195.

[13] C. H. Brans, J. Math. Phys. 6, 95 (1965).

[14] A. Karlhede, Gen. Rel. Grav. 12, 693 (1980).

[15] M. E. Araujo and J. E. F. Skea, Class. Quant. Grav. 5,
537-550 (1988).

[16] N. Kamran, Memoires Cl. Sci. Acad. Roy. Belg. 45, 121 (1989).

[17] A. Koutras, Class. Quant. Grav. 9, L143-L145 (1992).

[18] P. J. Olver, Equivalence, Invariants, and Symmetry (Cambridge University Press, Cambridge, 1995).

[19] J. E. F. Skea, Class. Quant. Grav. 14, 2947-2950 (1997).

[20] J. E. F. Skea, Class. Quant. Grav. 17, L69-L74 (2000).

[21] H. Stephani, D. Kramer, M. MacCallum, C. Hoenselaers, and E. Herlt, Exact Solutions of Einstein's Field Equations, 2nd ed. (Cambridge University Press, Cambridge, 2003).

[22] M. A. H. MacCallum, private communication (2008).

[23] R. Milson, A. Coley, V. Pravda, and A. Pravdova, Int. J. Geom. Methods Mod. Phys. 2, 41-61 (2005) arXiv:gr-qc/0401010.

[24] V. Pravda and A. Pravdova, Gen. Rel. Grav. 37, 12771287 (2005) arXiv:gr-qc/0501003.

[25] A. Coley, Class. Quant. Grav. 25: 033001 (2008) arXiv:0710.1598.

[26] H. Bondi, F. A. E. Pirani, and I. Robinson, Proc. Roy. Soc. London A 251, 519-533 (1959).

[27] J. Ehlers and W. Kundt, in Gravitation: An Introduction to Current Research, edited by L. Witten (Wiley, New York, 1962), pp. 49-101.

[28] M. A. H. MacCallum and J. E. F. Skea, in Algebraic Computing in General Relativity: Proceedings of the First Brazilian School on Computer Algebra vol. 2, edited by M. J. Rebouças and W. L. Roque (Oxford University Press, Oxford), pp. 1-172.

[29] C. W. Misner, K. S. Thorne, and J. A. Wheeler, Gravitation (W. H. Freeman, San Francisco, 1973).

[30] Peter Musgrave, Denis Pollney and Kayll Lake, GRTensorII Version 1.79 (R4), copyright 1994-2001 by the authors, latest version (2001 Feb. 6) available from $<$ http://grtensor.phy.queensu.ca/ $>$.

[31] A. S. Eddington, Nature 113, 192 (1924).

[32] D. Finkelstein, Phys. Rev. 110, 965-967 (1958).

[33] S. Chandrasekhar, The Mathematical Theory of Black Holes (Clarendon Press, Oxford, 1983).

[34] N. Pelavas, private communication (2008). 